\title{
Patrones de invasión de los pastizales de Uruguay a escala regional
}

\author{
Daniella Bresciano ${ }^{1, \otimes}$; Claudia Rodríguez ${ }^{2}$; Felipe Lezama $^{1} \&$ Alice $^{\text {Altesor }}{ }^{2}$ \\ 1. Unidad de Sistemas Ambientales, Facultad de Agronomía, Universidad de la República, Montevideo, Uruguay. 2. Instituto \\ de Ecología y Ciencias Ambientales, Facultad de Ciencias, Universidad de la República, Montevideo, Uruguay.
}

\begin{abstract}
Resumen. Los estudios sobre la relación entre la riqueza de especies nativas y exóticas en comunidades vegetales han generado evidencias opuestas, dependiendo, entre otros factores, de la escala espacial de análisis. En este trabajo se evaluó la invasión por especies exóticas de los pastizales naturales de Uruguay, a escala regional. Se analizaron 313 censos de vegetación correspondientes a cuatro regiones geomorfológicas: Cuesta Basáltica, Cuenca Sedimentaria, Centro Sur y Sierras del Este. Los resultados mostraron una relación negativa entre la riqueza de nativas y exóticas en Centro Sur, y entre la riqueza de nativas y la cobertura de exóticas en Centro Sur y Cuenca Sedimentaria. Cuando los análisis se realizaron por tipos funcionales, se observó que en la mayoría de las regiones la riqueza de las gramíneas $\mathrm{C} 4$ está asociada negativamente con las exóticas en su conjunto y fuertemente relacionada con la cobertura de Cynodon dactylon, la única especie exótica de este tipo funcional. Por el contrario, la riqueza de las especies C3 (tanto gramíneas como no-gramíneas) mostró un patrón irregular en las asociaciones con las exóticas. Además, la riqueza acumulada de exóticas se relacionó positivamente con la superficie bajo uso agrícola y el porcentaje de cobertura vegetal a escala regional. Nuestros resultados sugieren que si bien la presión de propágulos y la productividad regional favorecerían la introducción y establecimiento de las especies exóticas, el proceso de invasión en los pastizales naturales de Uruguay estaría en gran medida regulado por la composición funcional de la vegetación nativa, en particular por la presencia de gramíneas C4 perennes, que constituyen el grupo con mayor cobertura en estas comunidades. Esto podría explicar la baja incidencia de especies exóticas en los pastizales uruguayos.
\end{abstract}

[Palabras clave: especies exóticas, invasibilidad, gramíneas nativas C4, resistencia biótica, riqueza, tipos funcionales]

\begin{abstract}
AвSTRACT. Patterns of invasion in grasslands of Uruguay at a regional scale: Studies on the relationship between the richness of native and exotic plants have generated conflicting evidence depending, among others, on the spatial scale of analysis. This study assessed the invasion by exotic species in natural grasslands of Uruguay at a regional scale. We analyzed 313 vegetation census from four geomorphological regions: Cuesta Basaltica, Cuenca Sedimentaria, Centro Sur and Sierras del Este. The results showed that the relationship between native and exotic richness was negative for Centro Sur while the effect of native species richness on exotic plant cover was negative for Centro Sur and Cuenca Sedimentaria. In most analyses, the functional type C4 grasses was negatively associated with the exotic richness and cover, and strongly inhibits Cynodon dactylon, the only C4 exotic grass species present in the sites. Conversely, C3 species showed irregular patterns of association with the exotics. Also, exotic richness was positively related with the percentage of agricultural use and vegetation cover. Our results suggest that propagule pressure and productivity favour the introduction and establishment of exotic species, but they are largely controlled by the composition of the resident community, in particular by the presence of $\mathrm{C} 4$ perennial grasses, which are the group with the highest cover in these communities. This could explain the very low incidence of exotic species in Uruguayan grasslands.
\end{abstract}

[Keywords: exotic species, invasibility, native C4 grasses, biotic resistance, richness, functional types]

\section{INTRODUCCIÓN}

En la actualidad, las invasiones biológicas constituyen una de las causas principales de pérdida de biodiversidad a nivel mundial (Mack et al. 2000). Consisten en la expansión del rango de distribución de una especie en un hábitat donde no estaba antes presente. $\mathrm{Si}$ bien en sentido estricto es un fenómeno de carácter natural, su tasa de ocurrencia se ha incrementado de forma acelerada en el curso de la historia humana (Vitousek et al. 1996; Mack et al. 2000). La invasibilidad, entendida como la susceptibilidad del ambiente a la invasión de nuevas especies, es una propiedad emergente de los ecosistemas, potencialmente

$\triangle$ dbrescia@fagro.edu.uy afectada por varios factores que incluyen el clima local, las perturbaciones y las habilidades competitivas de las especies residentes (Lonsdale 1999). Además, el número de propágulos que arriban a una región (presión de propágulos) así como los atributos de las especies invasoras constituyen, junto con la invasibilidad, los tres factores principales que influyen en la invasión de un ambiente por nuevas especies (Davis et al. 2000).

Varias hipótesis y mecanismos han sido propuestos con el fin de explicar el éxito de la invasión de especies en los sistemas naturales (ver Catford et al. 2009). La hipótesis de diversidad-invasibilidad (Elton 1958) establece

Recibido: 9 de julio de 2013; Fin de arbitraje: 26 de julio; Última versión: 6 de septiembre; Aceptado: 19 de octubre 
que las comunidades más ricas en especies son menos susceptibles a ser invadidas debido principalmente al aumento de la "resistencia biótica", es decir, el conjunto de efectos negativos que ejercen las especies residentes sobre las especies invasoras (Levine et al. 2004). En general, se asume que la competencia es la principal fuerza reguladora de la invasión al limitar la disponibilidad de recursos en el ambiente para las invasoras (Davis et al. 2000; Hierro et al. 2005), si bien también se reconoce que la acción de depredadores, herbívoros y patógenos aumenta la resistencia de las comunidades a ser invadidas (Levine et al. 2004; Petermann et al. 2010).

La relación entre la riqueza de especies nativas y exóticas fue analizada en numerosas investigaciones, y ha generado evidencias controvertidas según la escala de análisis de los estudios; esto ha sido postulado como "la paradoja de la invasión" (Fridley et al. 2007). A escalas espaciales pequeñas $\left(<10 \mathrm{~m}^{2}\right)$, en general se ha observado una relación negativa entre estas variables. Por el contrario, a escalas espaciales grandes $\left(>1 \mathrm{~km}^{2}\right)$ generalmente se ha registrado una relación positiva (Lonsdale 1999; Fridley et al. 2007). Esta divergencia ha sido interpretada como consecuencia de los diferentes factores que operarían en las distintas escalas. A escalas pequeñas, los individuos podrían interactuar directamente entre sí y por lo tanto es esperable detectar los efectos de la competencia (Fridley et al. 2007). Esta interpretación se ajusta a la teoría del nicho, en donde las especies invasoras aprovechan los nichos vacantes y los recursos no utilizados por las especies residentes en comunidades no saturadas. Por el contrario, las relaciones positivas entre la riqueza de especies nativas y exóticas observadas a escalas espaciales mayores contradicen lo esperado por la teoría clásica de diversidadinvasibilidad y se han interpretado como evidencia de la importancia de factores tales como la presión de propágulos, las relaciones de facilitación, las perturbaciones y la heterogeneidad ambiental (Fridley et al. 2007).

Algunos de los factores que estarían operando a escalas grandes fueron incorporados en modelos que consideran que la supervivencia, crecimiento e interacciones entre las especies dependen de las propiedades del ambiente. Por ejemplo Huston (2004) plantea, en base al modelo de equilibrio dinámico (Huston 1979), que los procesos que influyen en el crecimiento y mortalidad de las especies nativas, en particular la productividad y las perturbaciones son los mismos que afectan los parámetros poblacionales de las especies exóticas. En consecuencia, las especies exóticas no difieren de manera cualitativa de las nativas, y la relación entre la riqueza de ambas será positiva a cualquier escala de análisis. Además, este modelo predice que el impacto de la invasión será mayor en los ambientes más productivos debido a que en éstos las tasas de crecimiento son altas, la diversidad es baja debido a la exclusión competitiva y las invasoras dominantes pueden potencialmente reemplazar a las especies nativas a partir de una perturbación.

Por otra parte, la paradoja de la invasión también puede analizarse a través de las hipótesis que prescinden del concepto de nicho, como la teoría neutral de la biodiversidad (Hubbell 2001). Esta teoría asume que las especies de un mismo nivel trófico son competitivamente idénticas y que el ensamblaje de las comunidades locales es un proceso azaroso guiado por la abundancia regional de propágulos y eventos demográficos estocásticos. En base a diversos estudios sobre invasiones, Daleo et al. (2009) señalan que esta teoría predice una relación positiva entre la riqueza de especies nativas y exóticas, tal como ha sido observado en los estudios a grandes escalas.

Otra predicción relacionada a la hipótesis clásica de diversidad-invasibilidad es que las especies residentes inhibirán más fuertemente el establecimiento de especies funcionalmente similares a ellas (i.e., hipótesis de la similitud límite o "limiting similarity"; Emery 2007; Catford et al. 2009), mientras que la teoría neutral predice un ensamblaje azaroso ya que considera a las especies funcionalmente equivalentes desde el punto de vista demográfico (Hubbell 2005). En este sentido, estudios experimentales apoyan la hipótesis clásica al observar que las especies pertenecientes al mismo grupo funcional que la especie exótica son más efectivas para limitar la invasión (Fargione et al. 2003; Petermann et al. 2010). Además, otros trabajos reportaron que ciertos grupos funcionales confieren mayor resistencia a la invasión. En particular, Fargione \& Tilman (2005) y Perelman et al. (2007) observaron que la biomasa y / o riqueza de las especies invasoras se correlaciona negativamente con la presencia de gramíneas $C 4$ residentes, evidenciando que este grupo funcional, que exhibe eficiencias elevadas en el uso de agua y nutrientes, es 
altamente competitivo e inhibe, además de las especies de su mismo grupo funcional, especies funcionalmente diferentes.

Los pastizales de Uruguay cubren $71 \%$ de la superficie del territorio (MGAP, DIEA 2001). Forman parte de los pastizales templados subhúmedos del Río de la Plata, que abarcan el centro-este de Argentina, todo el Uruguay y el sur de Brasil (Soriano 1992). Los pastizales uruguayos están dominados por gramíneas mayoritariamente con metabolismo fotosintético C4, acompañadas por hierbas y graminoides como especies subordinadas (Altesor et al. 2006).

En la región existen ciertos antecedentes de trabajos sobre especies invasoras en pastizales, mientras que en Uruguay los estudios son escasos. Recientemente, Fonseca et al. (2013) sintetizaron la información disponible sobre los registros de especies exóticas de los pastizales templados del Río de la Plata. Otros estudios más puntuales se han realizado en Brasil sobre la gramínea Eragrostis plana Nees, que ha invadido aproximadamente $10 \%$ del bioma en Río Grande (Medeiros \& Focht 2007). En la Pampa Inundable (Argentina) se analizaron los patrones de riqueza de especies nativas y exóticas, encontrándose casi $23 \%$ de especies exóticas (Chaneton et al. 2002; Perelman et al. 2007), en su mayoría hierbas anuales invernales. Este porcentaje contrasta con lo reportado por Lezama et al. (2006) para la Cuesta Basáltica de Uruguay, donde sólo se registró $4.3 \%$ de especies invasoras. Las diferencias en el grado de invasión de pastizales cercanos fitogeográficamente muestran que la invasión es un proceso multicausal y en ese sentido es un desafío hallar los mecanismos que explican estas diferencias.
En este trabajo describimos la composición y abundancia de la flora exótica en pastizales naturales uruguayos sometidos a pastoreo de las principales regiones geomorfológicas del país. Asimismo analizamos la relación entre la riqueza de especies nativas y la riqueza y cobertura de especies exóticas en los pastizales de estas regiones. De acuerdo a los antecedentes sería esperable encontrar una relación positiva entre estas variables, ya que a esta resolución espacial las interacciones bióticas pierden importancia en relación a otros factores extrínsecos (e.g., heterogeneidad ambiental, presión de propágulos) (Shea \& Chesson 2002; Davies et al. 2005). Sin embargo, la capacidad competitiva de las gramíneas C4 (Fargione \& Tilman 2005; Perelman et al. 2007), dominantes en estos pastizales, podría interferir en esta relación, generando resultados diferentes a lo esperado. Por ello se buscaron evidencias que apoyaran la hipótesis de la similitud límite como mecanismo complementario de la resistencia biótica, analizando por tipos funcionales las relaciones entre especies nativas y exóticas. En caso de operar la resistencia biótica, esperaríamos encontrar que los tipos funcionales nativos inhiban más fuertemente el establecimiento de especies funcionalmente similares a ellos.

\section{Métodos}

\section{Sitio de estudio}

El área de estudio abarca $116302 \mathrm{~km}^{2}$ (66\%) del territorio de Uruguay $\left(30^{\circ}\right.$ y $35^{\circ} \mathrm{S}, 53$ y $58^{\circ}$ $\mathrm{O})$, con alta proporción de pastizales naturales con uso pastoril. Comprende cuatro regiones geomorfológicas definidas por sus materiales geológicos, relieve, tipo de suelos y regionalización geográfica: Cuesta Basáltica, Centro Sur, Cuenca Sedimentaria y Sierras del Este (Panario 1988) (Figura 1, Tabla 1). Para la descripción de la heterogeneidad florística de los pastizales se

Tabla 1. Características generales de las cuatro regiones geomorfológicas.

Table 1. General characteristics of the four geomorphological regions.

\begin{tabular}{|c|c|c|c|c|}
\hline \multirow[t]{2}{*}{ Región } & \multirow{2}{*}{$\begin{array}{c}\text { ha } 10^{6} \\
\text { (\% de superficie } \\
\text { total del país) }\end{array}$} & \multicolumn{2}{|c|}{ Superficie relativa $(\%)^{*}$} & \multirow[t]{2}{*}{ Características generales del paisaje y suelo ${ }^{* *}$} \\
\hline & & $\begin{array}{l}\text { pastizales } \\
\text { naturales }\end{array}$ & uso agrícola & \\
\hline Cuesta Basáltica & $4.39(21)$ & 91.4 & 5.1 & Suelos superficiales y profundos. Sierras, colinas y lomadas \\
\hline $\begin{array}{l}\text { Cuenca } \\
\text { Sedimentaria }\end{array}$ & $2.52(14.3)$ & 69.3 & 21.5 & $\begin{array}{l}\text { Amplia variedad de materiales geológicos y suelos } \\
\text { asociados. Lomadas fuertes y colinas }\end{array}$ \\
\hline Centro Sur & $2.25(12.8)$ & 72.1 & 22.2 & Suelos superficiales y profundos. Relieve ondulado \\
\hline Sierras del Este & $2.52(14.3)$ & 87.6 & 1.2 & $\begin{array}{l}\text { Predominio de suelos superficiales y muy } \\
\text { superficiales, pedregosos. Relieve enérgico }\end{array}$ \\
\hline
\end{tabular}

*Baeza et al. (2011), según la cartografía de aproximadamente 70\% de la superficie total de las regiones.

**Millot et al. (1987). 
realizaron un total de 313 censos de la vegetación distribuidos en estas regiones geomorfológicas (Lezama et al. 2011). Los censos se realizaron en pastizales pastoreados, evitando áreas con alto grado de perturbación y signos de actividad agrícola. La vegetación se muestreó de acuerdo al método relevé (Mueller - Dombois \& Ellenberg 1974), en noviembre y diciembre del 2001 al 2009. La descripción del método de muestreo se detalla en Lezama et al. (2006) y Lezama et al. (2011); aquí se describen sólo algunos aspectos. Se utilizaron cuadrantes de $100 \mathrm{~m}^{2}$ que luego fueron ajustados a $25 \mathrm{~m}^{2}$ al verificar que esta superficie representaba el área mínima. Los cuadrantes fueron ubicados en el centro de áreas de vegetación homogénea ("stands"), donde se registraron todas las especies presentes. A cada especie se le asignó un valor de cobertura -abundancia siguiendo la escala de BraunBlanquet (1950). La lista de especies se completó luego de una amplia recorrida del stand por 10 minutos. De esta forma, se contó con 55 censos de la región Centro Sur, 70 de la Cuenca Sedimentaria, 80 de la Cuesta Basáltica y 108 de Sierras del Este. El conjunto de los 313 censos totalizaron un área de $13925 \mathrm{~m}^{2}$. La información se complementó con la identificación visual del porcentaje de cobertura vegetal por stand.

\section{Análisis de datos}

Se procesaron los datos de riqueza y cobertura de las especies nativas y exóticas para todos los censos de los cuatro inventarios fitosociológicos. Las especies registradas se asignaron a las categorías de tipos funcionales gramíneas y no-gramíneas (incluyen hierbas, graminoides y arbustos) con metabolismo fotosintético C3 y C4 (Rosengurtt 1979; Sage \& Monson 1999) y ciclo de vida anual y perenne (Rosengurtt 1979). Para cada región se calculó la diversidad alfa ( $\alpha=$ riqueza promedio de especies en los censos), la diversidad gama ( $\gamma=$ número de especies registradas en el conjunto de censos) y la diversidad beta $(\beta=\gamma / \alpha-1)$ de las especies nativas y exóticas (Whittaker 1972). Asimismo se calculó el valor de constancia de las especies exóticas en cada región, como la proporción de censos en los que apareció una especie en relación con el número total de censos realizados (Matteucci \& Colma 1982).

Para la comparación de la riqueza y cobertura promedio de especies exóticas entre regiones se aplicaron modelos lineales generalizados con función de enlace log, del paquete estadístico INFOSTAT con interfase en $\mathrm{R}$ (aplicación distribución libre Runner) al no cumplir con los supuestos de homogeneidad de varianzas y normalidad. Los datos de cobertura de las exóticas fueron transformados a clases de cobertura (números enteros entre 1 y 10) (Fridley 2013). Cuando los resultados fueron significativos se aplicaron pruebas de LSD Fisher $(\alpha=0.05)$, con ajuste por Bonferroni, para analizar las diferencias. Los datos de riqueza y cobertura de especies nativas cumplieron con los supuestos, y por tanto se aplicaron ANOVA's y pruebas a posteriori de LSD Fisher.

La relación entre la riqueza de especies nativas y la riqueza y cobertura de especies exóticas se analizó en cada región geomorfológica. Para los análisis

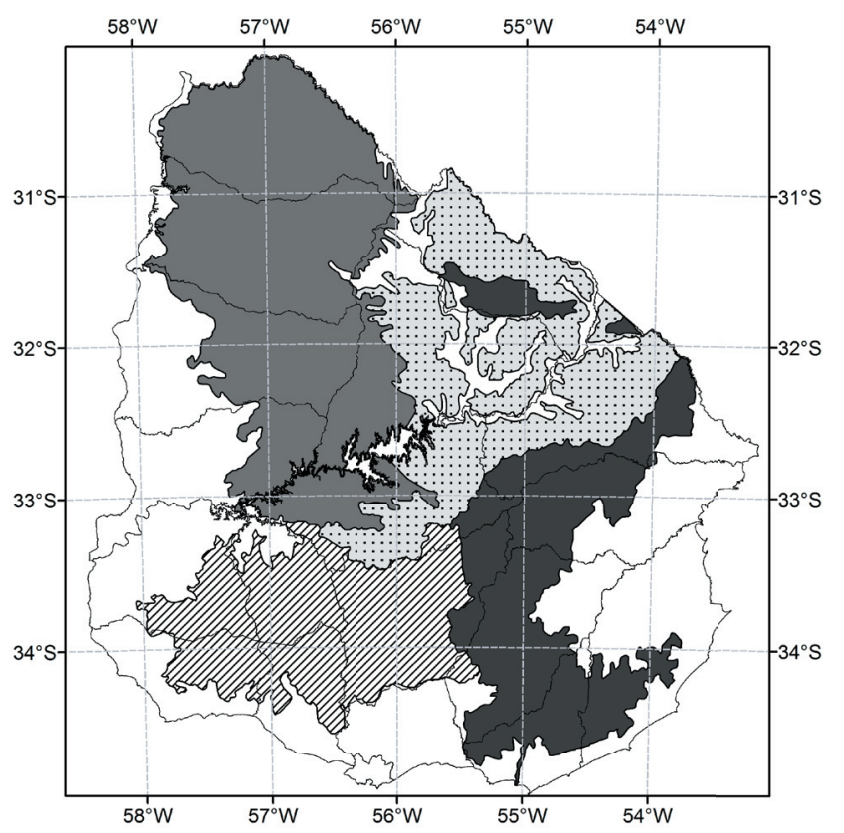

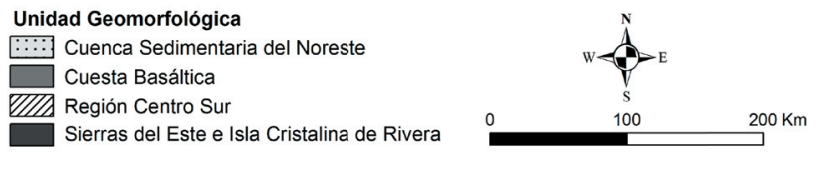

Figura 1. Regiones geomorfológicas de Uruguay donde se realizaron los censos (de Panario 1988).

Figure 1. Uruguayan geomorphological regions where census were conducted (from Panario 1988). 
se aplicaron modelos de regresión log lineal con distribución de Poisson [modelo $\log (\mu)=a+b x)$ ]. Los datos fueron corregidos por sobre-dispersión mediante el método de quasi-similitud (Crawley et al. 1999). Los mismos modelos fueron ajustados para analizar la relación entre los diferentes tipos funcionales de especies nativas y exóticas. Como Cynodon dactylon fue la única gramínea exótica C4 registrada en todas las regiones, para este tipo funcional analizamos únicamente la variable de cobertura.

Para explorar el efecto de factores extrínsecos sobre la invasión, se realizaron regresiones simples, utilizando como variable dependiente la diversidad gamma de exóticas de cada región. Las variables independientes utilizadas fueron: la diversidad beta de las especies nativas (como estimador de la heterogeneidad espacial; Davies et al. 2005), el porcentaje de uso agrícola del suelo (como estimador de la presión de propágulos) y la cobertura vegetal promedio (como estimador de la productividad). Se comprobó previamente el cumplimiento de los supuestos para este análisis.

\section{Resultados}

El número total de especies exóticas registrado fue de 49 ( $7 \%$ de la riqueza total). De éstas correspondieron 14 a la familia Poaceae y 35 a no-gramíneas pertenecientes a 14 familias, con predominio de la familia Asteraceae (13 especies). Las especies exóticas fueron en su mayoría de ciclo de vida anual con metabolismo C3 (82\%) (Apéndice, Información suplementaria). La cobertura promedio fue $4.7 \pm 0.7 \%$; de la cual $77.7 \%$ correspondió a gramíneas y $22.3 \%$ a no-gramíneas. Las especies que presentaron mayor porcentaje de cobertura fueron las gramíneas Cynodon dactylon $(2.8 \pm 0.6 \%)$ y Gaudinia fragilis $(1.5 \pm 0.3 \%)$. Lotus subbiflorus fue la especie que registró el mayor porcentaje de cobertura para el grupo de las no-gramíneas $(1.2 \pm 0.7 \%)$.

Asimismo, las regiones presentaron diferencias significativas tanto en riqueza promedio $\left(X^{2}=175.5, P<0.0001\right.$, g.1. $\left.=3\right)$ como en cobertura de especies exóticas $\left(X^{2}=753.3\right.$, $P<0.0001$, g.l. $=3$ ). Centro Sur fue la región más rica en exóticas y la que presentó el mayor valor de cobertura mientras que Cuesta Basáltica fue la región con menor riqueza y cobertura (Tabla 2). Por el contrario, para las especies nativas no se observaron diferencias en la riqueza promedio $(\mathrm{F}=1.5, P=0.22 ; \mathrm{g} .1 . \mathrm{l}=3)$, si bien la cobertura varió entre las regiones ( $\mathrm{F}=3.9, P=0.01$ g.l.=3) siendo Centro Sur y Cuenca Sedimentaria las regiones en donde se registraron los valores más altos (Tabla 2).

También se observó un escaso solapamiento funcional entre la flora exótica y nativa. Las exóticas de ciclo de vida anual con metabolismo fotosintético C3, tanto gramíneas como no-gramíneas, fueron mayoritarias en todas las regiones, mientras que en cobertura predominaron las gramíneas perennes $\mathrm{C} 4$ (C. dactylon) y las gramíneas anuales C3. En el caso de las nativas, en todas las regiones prevalecieron las especies perennes, en particular las gramíneas $\mathrm{C} 4$ y las no-gramíneas C3 (Figuras 2 y 3 ).

La relación entre la riqueza de especies nativas y de especies exóticas no fue significativa en la mayoría de las regiones geomorfológicas, excepto en la Región Centro Sur, donde se observó una relación negativa entre ambas variables. Por su parte, la relación entre la riqueza de nativas y la cobertura de exóticas fue negativa en la Cuenca Sedimentaria y Centro Sur, mientras que en las otras regiones no fue significativa (Tabla 3). También se detectó una relación negativa entre la riqueza de las gramíneas C4 nativas y la riqueza y cobertura de las especies exóticas en todas las regiones a excepción de la Cuesta Basáltica. Los otros tipos funcionales (gramíneas y no-gramíneas nativas C3) no mostraron asociaciones significativas con estas variables salvo en la región Centro Sur,

Tabla 2. Riqueza promedio (diversidad alfa), diversidad beta, riqueza total (diversidad gamma) y cobertura media ( \pm EE) de especies nativas y exóticas en cada región geomorfológica. Letras distintas representan diferencias significativas dentro de las columnas (prueba LSD $P<0.05$ ).

Table 2. Native and exotic species diversity (alpha, beta and gamma) and cover $( \pm \mathrm{SE})$ in the four geomorphological regions. Different letters indicate significant differences within columns (test LSD $P<0.05$ ).

\begin{tabular}{|c|c|c|c|c|c|c|c|c|c|}
\hline \multirow{2}{*}{ Regiones } & \multirow{2}{*}{ Especies exóticas (\%) } & \multicolumn{2}{|c|}{ Diversidad $\alpha$} & \multicolumn{2}{|c|}{ Cobertura (\%) } & \multicolumn{2}{|c|}{ Diversidad $\beta$} & \multicolumn{2}{|c|}{ Diversidad $\gamma$} \\
\hline & & Nativa & Exótica & Nativa & Exótica & Nativa & Exótica & Nativa & Exótica \\
\hline Cuesta Basáltica & $3.8 \pm 0.4 \mathrm{a}$ & $55.0 \pm 1.3 \mathrm{a}$ & $2.2 \pm 0.2 \mathrm{a}$ & $82.6 \pm 2.7 \mathrm{ab}$ & $0.6 \pm 0.2 \mathrm{a}$ & 6.5 & 11.7 & 350 & 14 \\
\hline $\begin{array}{l}\text { Cuenca } \\
\text { Sedimentaria }\end{array}$ & $6.7 \pm 0.4 b$ & $56.3 \pm 0.9 a$ & $4.0 \pm 0.2 b$ & $86.2 \pm 2.1 b$ & $3.8 \pm 1.2 b$ & 6.0 & 10.7 & 332 & 32 \\
\hline Centro Sur & $10.9 \pm 0.5 c$ & $53.3 \pm 1.3 a$ & $6.3 \pm 0.3 c$ & $87.1 \pm 2.8 b$ & $10.2 \pm 2.6 \mathrm{~d}$ & 5.4 & 7.2 & 284 & 38 \\
\hline Sierras del Este & $4.7 \pm 0.3 \mathrm{a}$ & $54.1 \pm 0.9 \mathrm{a}$ & $2.6 \pm 0.2 \mathrm{a}$ & $77.7 \pm 1.7 \mathrm{a}$ & $5.6 \pm 1.1 \mathrm{c}$ & 6.9 & 10.0 & 365 & 16 \\
\hline
\end{tabular}



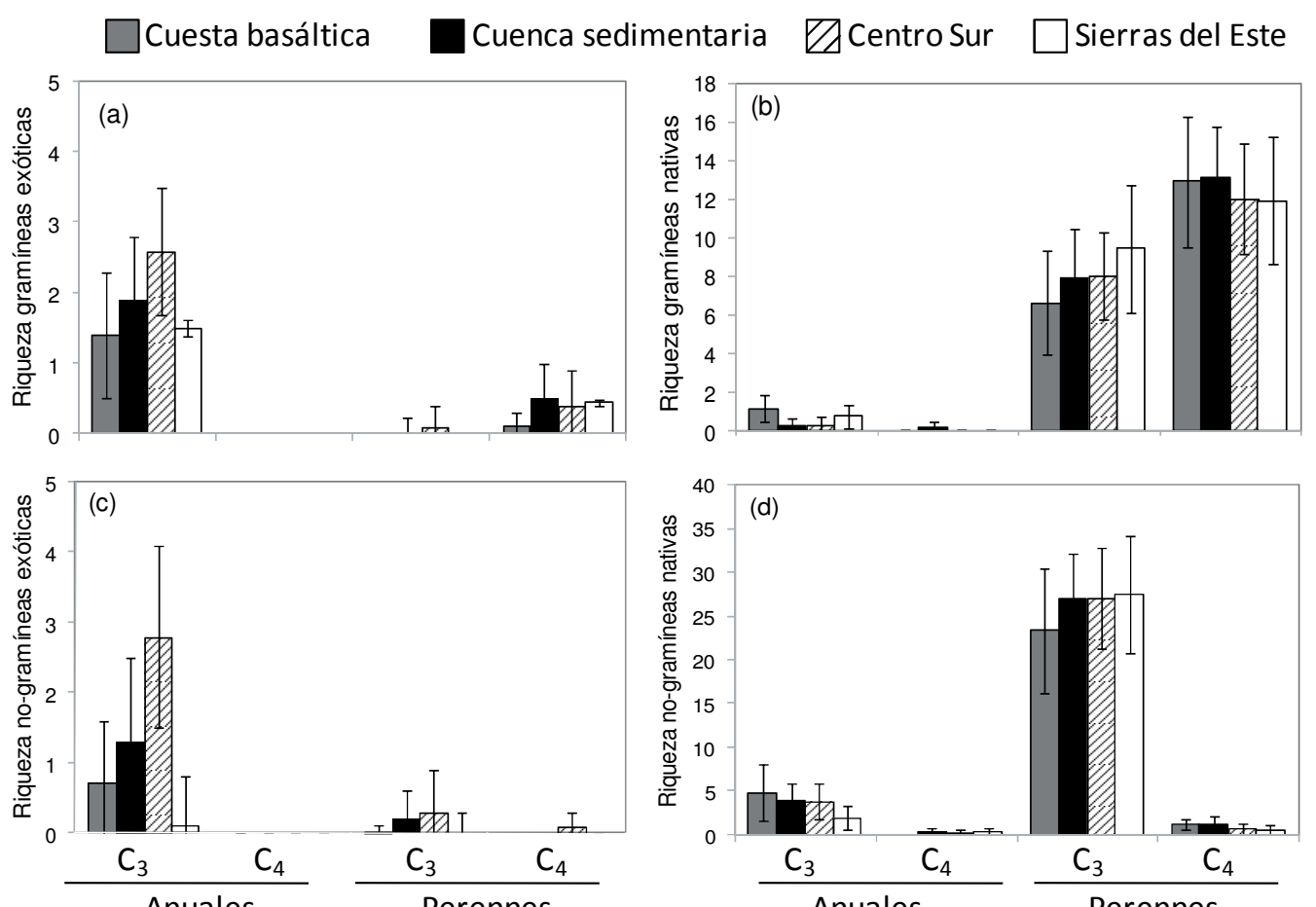

\section{Anuales Perennes}

Anuales

Perennes

Figura 2. Riqueza $( \pm E E)$ de gramíneas $(a, b)$ y no-gramíneas $(c, d)$ exóticas y nativas en las cuatro regiones geomorfológicas. Cada columna representa la riqueza promedio de especies por tipo funcional según metabolismo fotosintético $\left(\mathrm{C}_{3} \mathrm{oC}_{4}\right)$ y ciclo de vida (anual o perenne).

Figure 2. Species richness $( \pm \mathrm{SE})$ of exotic and native grasses $(a, b)$ and no-grasses $(c, d)$ in the four geomorphological regions. Each column represents the mean number of species by functional type according to photosynthetic pathway $\left(\mathrm{C}_{3}\right.$ or $\left.\mathrm{C}_{4}\right)$ and cycle (annual or perennial).

\section{$\square$ Cuesta basáltica $\square$ Cuenca sedimentaria $\square$ CentroSur $\square$ Sierras del Este}
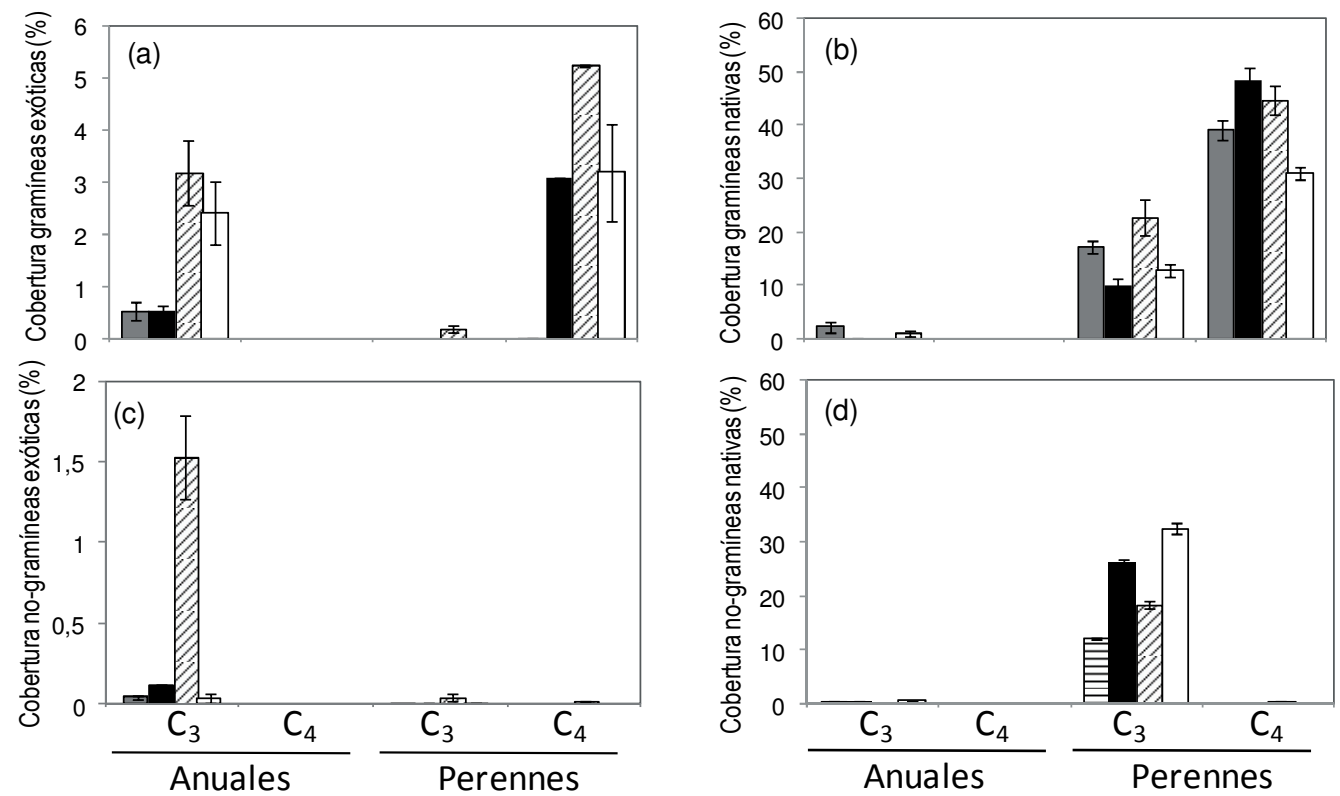

Figura 3. Cobertura $(\% \pm E E)$ de gramíneas $(a, b)$ y no-gramíneas $(c, d)$ exóticas y nativas en las cuatro regiones geomorfológicas. Cada columna representa el porcentaje de cobertura promedio de especies por tipo funcional según metabolismo fotosintético $\left(\mathrm{C}_{3} \mathrm{o} \mathrm{C}_{4}\right)$ y ciclo de vida (anual o perenne).

Figure 3. Cover $(\% \pm \mathrm{SE})$ of exotic and native grasses $(\mathrm{a}, \mathrm{b})$ and no-grasses $(\mathrm{c}, \mathrm{d})$ in the four geomorphological regions. Each column represents the mean cover by functional type according photosynthetic metabolism (C3 or C4) and life cycle (annual or perennial). 
Tabla 3. Resultados de las regresiones de Poisson entre la riqueza de especies nativas y la riqueza y cobertura de especies exóticas en cada región geomorfológica. En negrita valores significativos $(P \leq 0.05)$. GC4=gramíneas $C 4$; GC3=gramíneas C3; NGC3=no-gramíneas C3; CB=Cuesta Basáltica; $\mathrm{CS}=$ Cuenca Sedimentaria; CSur=Centro Sur, y $\mathrm{SE}=$ Sierras del Este. Const.=Constante; Est.=Estimador.

Table 3. Poisson regressions between native species richness and exotic richness and cover within each geomorphological region. Significant values $(P \leq 0.05)$ in bold. GC4 $=\mathrm{C} 4$ grasses; $\mathrm{GC} 3=\mathrm{C} 3$ grasses; NGC3 $=\mathrm{C} 3$ non-grasses. $\mathrm{CB}=$ Cuesta Basáltica; $\mathrm{CS}=$ Cuenca Sedimentaria; $\mathrm{CSur}=\mathrm{Centro}$ Sur, and $\mathrm{SE}=$ Sierras del Este. Const.=Constant; Est.=Estimator.

\begin{tabular}{|c|c|c|c|c|c|c|c|}
\hline \multirow[b]{2}{*}{ Región } & \multirow{2}{*}{$\begin{array}{c}\text { Riqueza } \\
\text { nativas }\end{array}$} & \multicolumn{3}{|c|}{ Riqueza Exóticas } & \multicolumn{3}{|c|}{ Cobertura Exóticas } \\
\hline & & Const. & Est. & P & Const. & Est. & $\mathrm{P}$ \\
\hline CB & Tod & 0.20 & 0.01 & 0.232 & & 0.005 & 0.412 \\
\hline \multirow{3}{*}{$(n=80)$} & & 1.20 & -0.03 & 0. & 0.4 & & 0.724 \\
\hline & & 0.4 & & & & & \\
\hline & & & & & & & 385 \\
\hline CS & & 1.67 & -0.01 & 372 & & & 003 \\
\hline \multirow{3}{*}{$(n=70)$} & & 2.50 & -0.08 & & 1.96 & & 001 \\
\hline & & & & & & & 531 \\
\hline & & 1.3 & & & & & 0.428 \\
\hline CSur & & 2.50 & -0. & 0.025 & 3.1 & -0.0 & $<0.001$ \\
\hline \multirow{3}{*}{$(n=55)$} & GC & 2.30 & -0.04 & 0.037 & 2.48 & -0. & $<0.001$ \\
\hline & & 2.1 & -0. & 0.08 & 1. & & $<0.001$ \\
\hline & & 2.2 & & & & & \\
\hline SE & Todas & 1.49 & -0. & 0.210 & 1.33 & -0.01 & 0.058 \\
\hline \multirow[t]{3}{*}{$(n=108)$} & & 1.46 & -0.04 & 0.014 & 1.44 & -0.08 & $<0.001$ \\
\hline & & 0.62 & & 0.224 & 0.0 & & 0.023 \\
\hline & NGC3 & 1.32 & -0.01 & 0.298 & 1.05 & -0.02 & 0.113 \\
\hline
\end{tabular}

donde las gramíneas nativas C3 se asociaron de manera negativa con la cobertura de exóticas y en la región Sierras del Este, donde esta asociación fue positiva (Tabla 3).

Los análisis de regresión entre tipos funcionales revelaron asociaciones negativas entre la riqueza de gramíneas $\mathrm{C} 4$ nativas y la cobertura dela única especie exótica deeste tipo funcional (C. dactylon) en todas las regiones. En la Cuesta Basáltica no fue posible realizar el análisis ya que $C$. dactylon fue registrada solo en un censo con $1 \%$ de cobertura, mientras que en los tres censos restantes el porcentaje fue menor a $1 \%$. También este tipo funcional nativo mostró asociaciones negativas con otros tipos funcionales exóticos en todas las regiones, pero siempre el efecto fue menor que con su propio tipo funcional. Por su parte, las gramíneas nativas C3 mostraron patrones irregulares. Sólo se observaron asociaciones negativas de este grupo con las exóticas de su mismo tipo funcional en Centro Sur (con la riqueza) y en Cuenca Sedimentaria (con la cobertura). En la región Centro Sur, el efecto inhibitorio sobre especies de otros tipos funcionales fue mayor que sobre su propio tipo funcional y en Sierras del Este las asociaciones fueron positivas. Las no- gramíneas nativas C3 sólo se relacionaron de forma negativa con las gramíneas C3 exóticas en la Cuenca Sedimentaria y en Centro Sur (Tabla 4).

En relación con los factores extrínsecos, la diversidad gamma de exóticas se asoció positivamente con el porcentaje de superficie agrícola $\left(\mathrm{R}^{2}=0.922, P=0.039\right)$ y con la cobertura vegetal promedio $\left(R^{2}=0.975, P=0.012\right)$, mientras que no se detectó una relación significativa con la diversidad beta de las especies nativas $\left(R^{2}=0.865, P=0.070\right)$ (Figura 4).
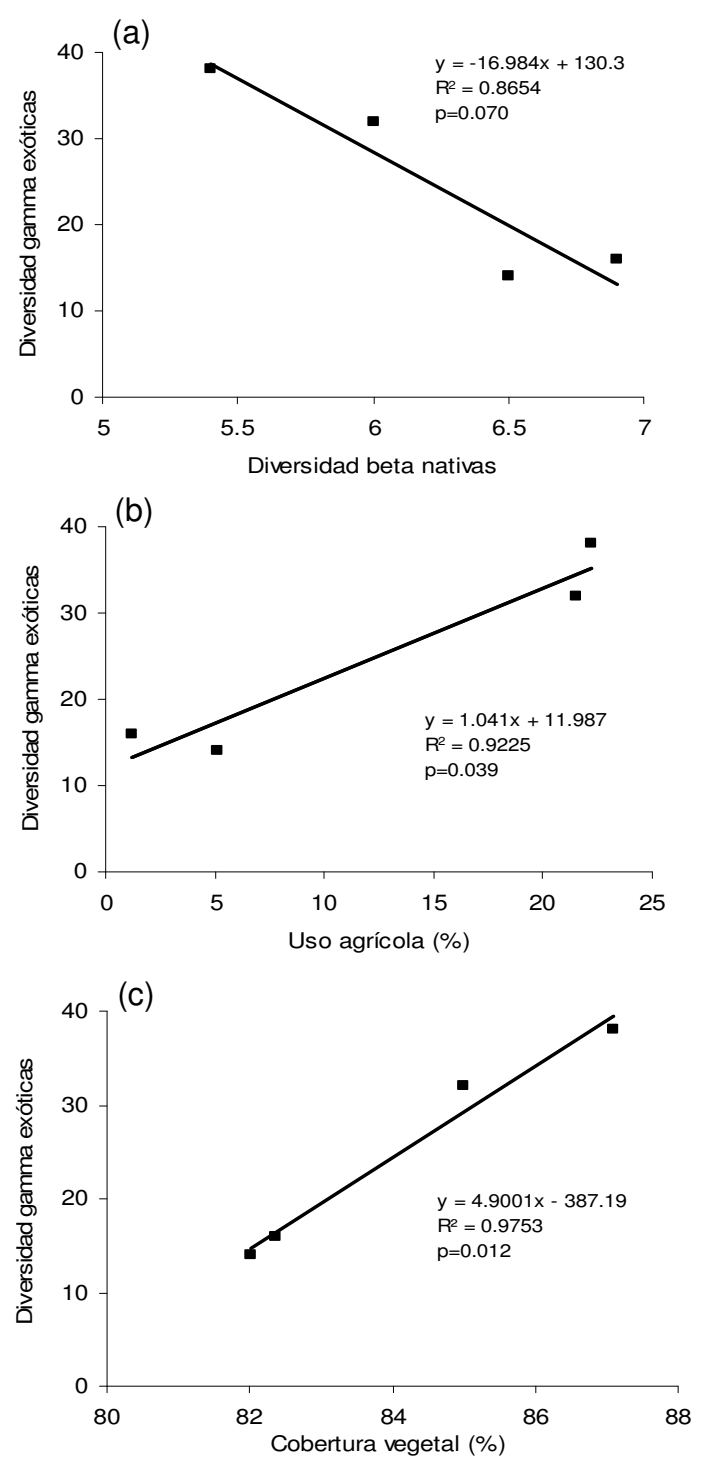

Figura 4. Relación entre la diversidad gamma de exóticas y (a) la diversidad beta de nativas, (b) el porcentaje de uso agrícola del suelo y (c) el porcentaje de cobertura vegetal de las cuatro regiones geomorfológicas.

Figure 4. Relationship of exotic gamma diversity with (a) native beta diversity, (b) percentage of agricultural land-use and (c) percentage of vegetation cover of the four geomorphological regions. 
Tabla 4. Relaciones entre la riqueza de tipos funcionales nativos y la riqueza y cobertura de tipos funcionales exóticos, estimadas por modelos de regresión de Poisson. Se muestra el valor del estimador (b). En negrita valores significativos $(P \leq 0.05)$. GC4=gramíneas $\mathrm{C} 4$; $\mathrm{GC} 3=$ gramíneas $\mathrm{C} 3 ; \mathrm{NGC} 3=$ no-gramíneas $\mathrm{C} 3 ; \mathrm{CB}=$ Cuesta Basáltica; CS=Cuenca Sedimentaria; CSur=Centro Sur, y $\mathrm{SE}=$ Sierras del Este.

Table 4. Relationship between richness of native functional types and richness and cover of exotic functional types. Values provided indicate parameter estimates from Poisson regression models. Significant values $(P \leq 0.05)$ in bold. $\mathrm{GC} 4=\mathrm{C} 4$ grasses; $\mathrm{GC} 3=\mathrm{C} 3$ grasses; $\mathrm{NGC} 3=\mathrm{C} 3$ nongrasses; $\mathrm{CB}=$ Cuesta Basáltica; $\mathrm{CS}=$ Cuenca Sedimentaria; CSur=Centro Sur, and SE=Sierras del Este.

\begin{tabular}{|c|c|c|c|c|c|c|}
\hline \multirow[b]{3}{*}{ Región } & \multirow{3}{*}{$\begin{array}{c}\text { TF } \\
\text { Nativos }\end{array}$} & \multicolumn{5}{|c|}{ TF exóticos } \\
\hline & & \multicolumn{2}{|c|}{ Riqueza † } & \multicolumn{3}{|c|}{ Cobertura } \\
\hline & & GC3 & NGC3 & GC4 & GC3 & NGC3 \\
\hline \multirow[t]{3}{*}{$\overline{C B}$} & GC4 & -0.02 & -0.02 & - & 0.01 & $-0.09 * * *$ \\
\hline & GC3 & 0.03 & 0.06 & - & 0.00 & 0.00 \\
\hline & NGC3 & 0.01 & 0.04 & - & 0.01 & -0.01 \\
\hline \multirow[t]{3}{*}{ CS } & GC4 & $-0.05^{*}$ & $-0.12^{* *}$ & $-0.14^{* * *}$ & -0.07 & 0.00 \\
\hline & GC3 & -0.00 & 0.02 & -0.02 & $-0.10^{*}$ & 0.00 \\
\hline & NGC3 & -0.00 & 0.02 & -0.01 & $-0.04^{*}$ & 0.03 \\
\hline \multirow[t]{3}{*}{ CSur } & GC4 & -0.01 & -0.04 & $-0.18^{* * *}$ & -0.03 & $-0.12^{* * *}$ \\
\hline & GC3 & $-0.01 *$ & $-0.08^{* *}$ & $-0.18^{* * *}$ & -0.03 & $-0.12^{* *}$ \\
\hline & NGC3 & $-0.02^{*}$ & -0.00 & -0.01 & $-0.04^{* *}$ & 0.01 \\
\hline \multirow[t]{3}{*}{ SE } & GC4 & -0.00 & -0.08 & $-0.11^{* * *}$ & 0.03 & $-0.03^{*}$ \\
\hline & GC3 & 0.02 & -0.04 & $0.05^{*}$ & 0.02 & $0.03^{*}$ \\
\hline & NGC3 & -0.01 & -0.02 & -0.01 & -0.01 & 0.00 \\
\hline
\end{tabular}

*: $P<0.05 ; * *: P<0.01 ; * * *: P<0.001$

+ Ausencia de valores en GC4 debido a $n=1$

\section{DISCUSIÓN}

El relevamiento de una porción amplia del territorio uruguayo para describir la heterogeneidad de los pastizales naturales reveló tres aspectos interesantes en relación con la invasión de especies. Los mismos distinguen a estos pastizales dentro de la región y son: la baja incidencia de especies exóticas, la existencia de patrones sugiriendo resistencia biótica a la invasión a escala regional y la importancia de las gramíneas $\mathrm{C} 4$ nativas como el tipo funcional más efectivo para controlar esta invasión.

La invasión por especies exóticas fue en general limitada, tanto en términos de riqueza como de cobertura, ¿Qué mecanismos promoverían la invasión, si bien escasa, de estos pastizales? Por una parte, nuestros resultados muestran un escaso solapamiento entre la composición funcional de la flora nativa y exótica en las cuatro regiones geomorfológicas analizadas. La flora nativa está compuesta principalmente por especies perennes (tanto gramíneas como no-gramíneas), mientras que las especies exóticas son en su mayoría hierbas anuales C3. Las plantas exóticas pertenecientes a tipos funcionales diferentes a los dominantes en la comunidad nativa tendrían ventajas para establecerse, debido probablemente a la existencia de recursos no usados o subutilizados por parte de las especies residentes (Davis et al. 2000; Hierro et al. 2005), generando complementariedad en el uso de los recursos (Fargione \& Tilman 2005). Por otra parte, la disponibilidad de propágulos provenientes de sitios bajo uso agrícola provocaría un aumento en la invasión por exóticas ( $\mathrm{D}^{\prime}$ Antonio et al. 2001; Haretche \& Rodríguez 2004). El porcentaje de suelo destinado a la agricultura y la riqueza total de exóticas en las regiones estuvieron directamente relacionados. La Cuesta Basáltica y Sierras del Este fueron las regiones con menor riqueza de plantas exóticas y son las que presentan el menor porcentaje de transformación en el uso del suelo, por lo que la presión de propágulos sería baja. Por su parte Centro Sur y Cuenca Sedimentaria, con más de $20 \%$ de superficie bajo uso agrícola, son las regiones más ricas en exóticas. Además, la relación positiva encontrada entre la cobertura vegetal y la riqueza total de exóticas de cada región sugiere que la productividad podría ser otro factor que favorece la invasión. En este sentido, Cuenca Sedimentaria y particularmente Centro Sur contarían con mayor disponibilidad de recursos y por lo tanto menores restricciones para el establecimiento y desarrollo de las especies exóticas que arriben al área (Huston 2004).

Por último, la ausencia de relación entre la heterogeneidad espacial en la composición de especies nativas (diversidad beta) y la riqueza total de exóticas contradice lo esperado por la teoría, en relación a que los ambientes más heterogéneos permiten una mayor coexistencia de especies y difiere de las asociaciones positivas observadas en otras investigaciones (Shea \& Chesson 2002; Davies et al. 2005). En nuestro estudio, el rango de variación de la diversidad beta de las regiones es pequeño en comparación con otros trabajos donde utilizan esta variable como descriptora de la heterogeneidad ambiental (e.g., Davies et al. 2005). Esto podría deberse a que las cuatro regiones están sometidas a una presión de pastoreo relativamente uniforme que actúa como factor generador de homogeneidad espacial, tal como fue reportado por Lezama et al. (2013) para los pastizales del Río de la Plata.

Los mecanismos discutidos sugieren que la composición funcional de las invasoras, la presión de propágulos y la productividad 
podrían estar afectando la magnitud de la invasión de estas regiones, pero no explicarían la baja incidencia de exóticas en el territorio.

Con el fin de explorar si la comunidad receptora actúa como barrera a la invasión, buscamos evidencias compatibles con la hipótesis de resistencia biótica. La relación entre la riqueza de especies nativas y la riqueza y/o cobertura de exóticas fue negativa para las regiones Centro-Sur y Cuenca Sedimentaria y no significativa para la Cuesta Basáltica y Sierras del Este. Las relaciones negativas entre estas variables constituyen un resultado no esperado de acuerdo a nuestra hipótesis de trabajo y son consistentes con la predicción del modelo clásico de invasión (Elton 1958) que establece que la riqueza de la comunidad nativa afecta de forma negativa a las exóticas, fundamentalmente a través de las interacciones de competencia que se establecen entre ellas. Sin embargo, a escalas espaciales mayores a $1 \mathrm{~km}^{2}$ por lo general se observa una relación positiva entre especies nativas y exóticas, lo que ha sido interpretado como una consecuencia del aumento en la variación de las condiciones ambientales y la dilución de los efectos de la competencia (Fridley et al. 2007; Perelman et al. 2007; Souza et al. 2011). Este patrón también es el esperado bajo los supuestos de las hipótesis que prescinden de las interacciones interespecíficas para explicar el fenómeno de la invasión, como el modelo de equilibrio dinámico de Huston (2004) o la teoría neutral de Hubbell (2001). Por lo tanto, las relaciones negativas observadas en nuestro trabajo constituyen un patrón poco frecuente en los estudios sobre invasiones realizados a estas escalas y sugieren que los mecanismos que determinan estas relaciones pueden variar entre sistemas, dependiendo del contexto (Souza et al. 2011).

Por otra parte, la relación negativa entre la riqueza de especies nativas y la cobertura de especies exóticas podría deberse a que las comunidades residentes, más que impedir el establecimiento de las exóticas, actuarían como barrera para su propagación, impidiendo que alcancen valores altos de cobertura. Esta interpretación se ajusta a lo planteado por Levine et al. (2004), quienes dividen el proceso de invasión en cuatro fases: introducción, establecimiento, propagación e impacto. Según estos autores, la hipótesis de Elton (1958) sugiere que la resistencia biótica debería operar más fuertemente en la fase de establecimiento. Sin embargo, a partir de los resultados de un meta-análisis que incluye varios estudios en comunidades vegetales, los autores postulan que las comunidades receptoras actúan posteriormente en el proceso de invasión (en las fases de propagación e impacto), estableciendo un efecto regulador o de contención, más que de resistencia a la invasión.

Nuestros resultados apoyan la hipótesis de la similitud límite como mecanismo complementario de la resistencia biótica solamente para el tipo funcional de gramíneas C4. En todas las regiones (a excepción de la Cuesta Basáltica donde no fue posible realizar el análisis), el mayor impacto negativo de las gramíneas nativas $\mathrm{C} 4$ se registró sobre la cobertura de $C$. dactylon (única gramínea C4 exótica) y también mostró asociaciones negativas con otros tipos funcionales exóticos. Por otro lado, fue el único tipo funcional que consistentemente exhibió relaciones negativas con la riqueza y cobertura total de exóticas. Estos resultados coinciden con el patrón observado en otros estudios (e.g., Smith \& Knapp 1999; Fargione et al. 2003; Fargione \& Tilman 2005; Perelman et al. 2007) que mostraron el fuerte efecto inhibidor que ejerce este tipo funcional en la invasión. Las gramíneas C4 son el tipo funcional con mayor cobertura en los pastizales uruguayos sometidos a pastoreo (Rodríguez et al. 2003; Altesor et al. 2006). Su dominancia podría explicarse por sus elevadas tasas de crecimiento relativo, tasas de macollaje y de producción de hojas (Leoni et al. 2009). Además de limitar la invasión de especies de su mismo tipo funcional, el dosel más cerrado de las gramíneas C4 luego de su período de crecimiento a finales del verano, también podría limitar el establecimiento y propagación del conjunto de exóticas, mayoritariamente anuales C3, cuyo reclutamiento ocurre principalmente durante el fin del verano o a principios de otoño (Perelman et al. 2007). Por su parte, las gramíneas nativas con metabolismo fotosintético C3 mostraron un patrón irregular de asociación con las exóticas. En la región Centro Sur, el efecto inhibitorio de este grupo de nativas fue mayor sobre especies de otros tipos funcionales exóticos que sobre su propio tipo funcional mientras que en Sierras del Este estas asociaciones fueron positivas. Las no-gramíneas nativas C3 mostraron pocas asociaciones negativas, $\mathrm{y}$ en todos los casos fue con las gramíneas exóticas C3. Estos resultados indican que la hipótesis de similitud límite no se aplica para los grupos funcionales con metabolismo fotosintético C3. Además, no se observaron relaciones significativas entre estos grupos y la 
riqueza total de exóticas, y salvo en una región, tampoco hubo relación con la cobertura, lo que reflejaría una menor capacidad de las especies C3 para inhibir la invasión.

En comparación con la Pampa Inundable en Argentina, aunque existe un predominio de plantas exóticas C3 de ciclo anual en ambos países, el porcentaje de especies exóticas en Uruguay es sensiblemente menor (7\%) que el registrado en la Pampa Inundable (23\%) (Perelman et al. 2007) y las relaciones entre especies nativas y exóticas son de signos opuestos (negativa en los pastizales uruguayos y positiva en la Pampa Inundable). Nuestros resultados sugieren que el predominio de gramíneas C4 confiere resistencia biótica a la invasión de los pastizales uruguayos, mientras que en la Pampa Inundable, según Perelman et al. (2007), el proceso de invasión estaría regulado principalmente por factores abióticos y el tamaño del pool regional de especies excepto en las praderas húmedas con mayor cobertura de gramíneas C4. Para lograr una comprensión integral del proceso de invasión en ambos países deberían incorporarse otras variables tales como la tasa e intensidad de transformación del uso de la tierra y la historia de uso de los pastizales (Paruelo et al. 2006; Blum et al. 2008). Los procesos subyacentes asociados a estas variables pueden aportar más información para comprender la diferencia entre los patrones a grandes escalas en ambos países.

En síntesis, nuestros resultados indican que la resistencia biótica estaría operando en la mayor parte de los pastizales naturales de Uruguay a escala regional, principalmente debido a la presencia de gramíneas $C 4$ perennes, que constituyen el grupo con mayor cobertura en estas comunidades. Asimismo identificamos ciertos factores ambientales, como la presión de propágulos y la productividad a nivel regional, que estarían favoreciendo la invasión de los pastizales. En este mismo sentido, en un reciente estudio a escala mundial, Seabloom et al. (2013) encontraron que los principales predictores de la invasión de pastizales eran la riqueza de gramíneas nativas y la proximidad de áreas cultivadas. Sin embargo la susceptibilidad de las comunidades a la invasión no es un atributo estático o permanente, sino una propiedad emergente de cada una de las comunidades o ecosistemas (Davis et al. 2000). Esto significaría que si las actuales condiciones varían, debido al aumento e intensificación del uso del suelo, el escenario de las invasiones podría ser menos promisorio para nuestros pastizales.
Agradecimientos: Agradecemos a B. Costa por sus valiosos comentarios al manuscrito y A. Borges por su asesoramiento estadístico. Tres revisores anónimos aportaron numerosas sugerencias para mejorar el trabajo. D. Bresciano recibió financiación de la Agencia Nacional de Investigación e Innovación (ANII) para la realización de su doctorado.

\section{BiBLIOGRAFÍA}

Altesor, A; G PiñeIro; F LeZama; RB Jackson; M Sarasola; ET AL. 2006. Ecosystem changes associated with grazing in subhumid South American grasslands. J. Veg. Sci., 17:323-332.

Baeza, S; F Gallego; F Lezama; A Altesor \& JM Paruelo. 2011. Cartografía de los pastizales naturales en las regiones geomorfológicas de Uruguay predominantemente ganaderas. Pp. 33-54 en: Altesor, A; W Ayala; JM Paruelo, JM (eds.). Bases ecológicas y tecnológicas para el manejo de pastizales. Serie FPTA INIA 26, Uruguay.

Blum, A; I Narbondo; G Oyhantcabal \& D Sancho. 2008. Soja transgénica y sus impactos en Uruguay. La nueva colonización. Ed. M.I. Cárcamo. RAPAL - Uruguay.

Braun Blanquet, J. 1950. Sociología vegetal. ACME. Buenos Aires.

CAtford, J; R Jansson \& C Nilsson. 2009. Reducing redundancy in invasion ecology by integrating hypotheses into a single theoretical framework. Diversity Distrib., 15:22-40.

Chaneton, EJ; SB Perelman; M Omacini \& RJC León. 2002. Grazing, environmental heterogeneity, and alien plant invasions in temperate Pampa grasslands. Biol. Invasions, 4:7-24.

Crawley, MJ; SL Brown; MS Heard \& GR Edwards. 1999. Invasion-resistance in experimental grassland communities: species richness or species identity? Ecol. Lett., 2:140-148.

Daleo, P; J Alberti \& O Iribarne. 2009. Biological invasions and the neutral theory. Diversity Distrib., 15:547-553.

D'Antonio, C; J Levine \& M Thomsen. 2001. Ecosystem resistance to invasion and the role of propagule supply: a California perspective. J. Med. Ecol., 2:233-245.

Davies, KF; P Chesson; S Harrison; BD Inouye; BA Melobourne; ET AL. 2005. Spatial heterogeneity explains the scale dependence of the native-exotic diversity relationship. Ecology, 86:1602-1610.

Davis, MA; JP Grime \& K Thompson. 2000. Fluctuation resources in plant communities: a general theory of invasibility. J. Ecol., 88:528-534.

Elton, CS. 1958. The ecology of invasions by animals and plants. Methuen, London, UK.

EMERY, SM. 2007. Limiting similarity between invaders and dominant species in herbaceous plant communities? J. Ecol., 95:1027-1035.

FARGIONE, J \& D TILMAN. 2005. Diversity decreases invasion via both sampling and complementarity effects. Ecol. Lett., 8:604-611.

Fargione, J; CS Brown \& D Tilman. 2003. Community assembly and invasion: an experimental test of neutral versus niche processes. PNAS, 100:8916-8920.

Fonseca, CR; DL Guadagnin; C Emer; S Masciandri; P GermaIN; ET AL. 2013. Invasive alien plants in the Pampas grasslands; a tri-national cooperation challange. Biol. Invas., doi: 10.1007/s10530-013-0406-2.

FRIDLEY, JD. 2013. Generalized linear models in R. http: //plantecology.syr.edu/fridley/bio793/glm.html. 
Acceso: 5 de agosto de 2013.

Fridley, JD; JJ Stachowicz; S Naeem; DF Sax; W Seabloom; ET AL. 2007. The invasion paradox: reconciling pattern and process in species invasions. Ecology, 88:3-17.

Haretche, F \& C Rodríguez. 2004. Banco de semillas de un pastizal uruguayo bajo diferentes condiciones de pastoreo. Ecología Austral, 16:105-113.

Hierro, JL; JL Maron \& RM Callaway 2005. A biogeographical approach to plant invasions: the importance of studying exotics in their introduced and native range. J. Ecol., 93:5-15.

HubBelL, ST. 2005. Neutral theory in community ecology and the hypothesis of functional equivalence. Funct. Ecol., 19:166-172.

HubBell SP. 2001. The Unified Neutral Theory of Biodiversity and Biogeography. Princeton University Press, New Jersey.

Huston, M. 2004. Management strategies for plant invasions: manipulating productivity, disturbance, and competition, Diversity Distrib., 10:167-178

Huston, MA. 1979. A general hypothesis of species diversity. Am. Nat., 113:81-101.

Leoni, E; A Altesor \& Ja Paruelo. 2009. Explaining patterns of production from individual level traits. J. Veg. Sci., 20:612-619.

LeVINE, JM; PB AdLer \& SG YelenIK. 2004. A meta-analysis of biotic resistance to exotic plant invasions. Ecol. Lett., 7:975-989.

Lezama, F; S Baeza; A Altesor; A Cesa; EJ Chaneton; et al. 2013. Variation of grazing-induced vegetation changes across a large-scale productivity gradient. J. Veg. Sci., Doi:10.1111/jvs.12053.

Lezama, F; A Altesor; M Pereira \& JM Paruelo. 2011. Descripción de la heterogeneidad florística de las principales regiones geomorfológicas de Uruguay. Pp.15-32 en: Altesor, A; W Ayala \& JM Paruelo (eds.). Bases ecológicas y tecnológicas para el manejo de pastizales. Serie FPTA - INIA 26. Uruguay.

Lezama, F; A Altesor; RJ León \& JM Paruelo. 2006. Heterogeneidad de la vegetación en pastizales naturales de la región basáltica de Uruguay. Ecología Austral, 16: 167-182.

LONSDALE, WP. 1999. Global patterns of plant invasions and the concept of invasibility. Ecology, 8:1522-1536.

MaCK, RN; D SimberlofF; WM Lonsdale; H Evans; M Clout; ET AL. 2000. Biotic invasions: Causes, epidemiology, global consequences, and control. Ecol. Appl., 10:689710.

Matteucci, S \& A Colma. 1982. Metodología para el estudio de la vegetación. Monografía OEA, Washington, DC. Pp. 53-54.

Medeiros, RB \& T Focht. 2007. Invasão, prevenção, controle e utilização do capim-annoni 2 (Eragrostis plana Nees) no Rio Grande do Sul, Brasil. Revista Agropecuária Gaúcha, 13:105-114.

MGAP, DIEA. 2001. Censo General Agropecuario. 2000. http: //www.mgap.gub.uy/Dieaanterior/CENSOVOL2/ indice.htm.

Millot, JC; D Risso \& R Methol. 1987. Relevamiento de pasturas naturales y mejoramientos extensivos en áreas ganaderas del Uruguay. Informe Técnico, Ministerio de Ganadería, Agricultura y Pesca. Montevideo. Uruguay.

Mueller-Dombois, D\& H Ellenberg. 1974. Aim and methods of vegetation ecology. John Wiley, Nueva York.

PANARIO, D. 1988. Geomorfología del Uruguay. Facultad de Humanidades y Ciencias, Universidad de la República. Montevideo, Uruguay.

Paruelo, JM; JP Guerschman; G Piñeiro; EG Jobbágy; SR Verón; ET AL. 2006. Cambios en el uso de la tierra en Argentina y Uruguay: marcos conceptuales para su análisis. Agrociencia, 10:47-61.

Perelman, SB; WB Batista; E Chaneton \& RJC León. 2007. Habitat stress, species pool size, and biotic resistance influence exotic plant richness in the Flooding Pampa grasslands. J. Ecol., 95:662-673.

Petermann, JS; AJF Fergus; C Roscher; LA Turnbull; A Weigelt; ET AL. 2010. Biology, chance, or history? The predictible reassembly of temperate grassland communities. Ecology, 91:408-421.

Rodríguez, C; E Leoni; F Lezama \& A Altesor. 2003. Temporal trends in species composition and plant traits in natural grasslands of Uruguay. J. Veg. Sci., 14: 433-440.

RosengurTt, B. 1979 Tablas de comportamiento de las especies de plantas de campos naturales en el Uruguay. Publicación de la Facultad de Agronomía. Montevideo, Uruguay.

SAGE, RF; M Li \& RK Monson. 1999. The taxonomic distribution of C4 photosynthesis. Pp. 551-581 en: Sage, RF \& RK Monson (eds.). C4 plant biology. Academic Press.

SeAbloOm, E; ET Borer; Y Buckley; WE Cleland; K Davies; ET AL. 2013. Predicting invasion in grassland ecosystems: is exotic dominance the real embarrassment of richness? Glob. Change Biol., doi: 10.1111/gcb.12370.

SHEA, K \& P CHESSON. 2002. Community ecology theory as a framework for biological invasions. Trends Ecol. Evol., 17:170-176.

SMITH, MD \& AK KNAPr. 1999. Exotic plant species in a C4-dominated grassland: invasibility, disturbance, and community structure. Oecologia, 120:605-612.

Soriano, A. 1992. Río de la Plata grasslands. Pp. 367-407 en: Coupland, RT (ed.). Natural grasslands. Introduction and western hemisphere. Elsevier.

Souza, L; WA BunN; D Simberloff; RM Lawton \& NJ SANDERS. 2011. Biotic and abiotic influences on native and exotic richness relationship across spatial scales: favourable environments for native species are higly invasible. Funct. Ecol., 25:1106-1112.

Vitousek, PM; CM D'Antonio; LL Loope \& R Westbrooks. 1996. Biological invasions as global environmental change. Am. Sci., 84:468-478.

Whitakker, RH. 1972. Evolution and measurement of species diversity. Taxon, 21:213-251. 


\section{INFORMACIÓN SUPLEMENTARIA}

Apéndice. Especies exóticas registradas en 313 censos ubicados en las cuatro regiones geomorfológicas con mayor porcentaje de pastizales naturales de Uruguay. $\mathrm{A}=$ anual; $\mathrm{B}=$ bianual; $\mathrm{P}=$ perenne; $\mathrm{CB}=\mathrm{Cuesta}$ Basáltica; $\mathrm{CS}=\mathrm{Cuenca}$ Sedimentaria; CSur=Centro Sur, y SE=Sierras del Este.

Appendix. Exotic species recorded in 313 census located in the four geomorphological regions with higher percentage of natural grasslands of Uruguay. $\mathrm{A}=$ annual; $\mathrm{B}=$ biennial; $\mathrm{P}=$ perennial; $\mathrm{CB}=\mathrm{Cuesta}$ Basáltica; $\mathrm{CS}=\mathrm{Cuenca}$ Sedimentaria; CSur=Centro Sur, and SE=Sierras del Este.

\begin{tabular}{|c|c|c|c|c|c|c|c|}
\hline \multirow{2}{*}{$\begin{array}{l}\text { Especies } \\
\text { Gramíneas }\end{array}$} & \multirow[t]{2}{*}{ Familia } & \multirow[t]{2}{*}{$\begin{array}{l}\text { Ciclo de } \\
\text { vida }\end{array}$} & \multirow[t]{2}{*}{$\begin{array}{l}\text { Metabolismo } \\
\text { fotosintético }\end{array}$} & \multicolumn{4}{|c|}{$\begin{array}{c}\text { Constancia por región } \\
(\%)\end{array}$} \\
\hline & & & & $\mathrm{CB}$ & CS & CSur & SE \\
\hline Aira caryophyllea L. & Poaceae & A & $\mathrm{C} 3$ & & 7 & & 3 \\
\hline Aira elegans Willd & Poaceae & A & $\mathrm{C} 3$ & & 51 & 33 & 32 \\
\hline Briza maxima $\mathrm{L}$. & Poaceae & A & $\mathrm{C} 3$ & & & 2 & \\
\hline Briza minor $\mathrm{L}$ & Poaceae & A & $\mathrm{C} 3$ & 50 & 86 & 84 & 46 \\
\hline Bromus commutatus Schrad. & Poaceae & A & $\mathrm{C} 3$ & & & 6 & \\
\hline Bromus hordeaceus L. & Poaceae & A & $\mathrm{C} 3$ & & & 16 & \\
\hline Cynodon dactylon (L.) Pers. & Poaceae & $\mathrm{P}$ & $\mathrm{C} 4$ & 5 & 49 & 42 & 44 \\
\hline Dactylis glomerata $\mathrm{L}$. & Poaceae & $\mathrm{P}$ & $\mathrm{C} 3$ & & & 2 & \\
\hline Festuca arundinacea Schreb. & Poaceae & $\mathrm{P}$ & $\mathrm{C} 3$ & & 3 & 2 & \\
\hline Gaudinia fragilis (L.) P. Beauv. & Poaceae & A & $\mathrm{C} 3$ & & 17 & 40 & 52 \\
\hline Holcus lanatus L. & Poaceae & $\mathrm{P}$ & $\mathrm{C} 3$ & & & 4 & \\
\hline Koeleria phleoides (Villars) Pers. & Poaceae & A & $\mathrm{C} 3$ & 40 & 6 & 7 & \\
\hline Lolium multiflorum Lam. & Poaceae & A & $\mathrm{C} 3$ & 36 & 21 & 69 & 14 \\
\hline Роа аппиа $\mathrm{L}$. & Poaceae & A & $\mathrm{C} 3$ & 13 & 6 & & 6 \\
\hline \multicolumn{8}{|l|}{ No gramíneas } \\
\hline Ammi majus L. & Apiaceae & A & $\mathrm{C} 3$ & & & 2 & \\
\hline Anagallis arvensis L. & Acanthaceae & $\mathrm{A}$ & $\mathrm{C} 3$ & & 1 & 16 & \\
\hline Anthemis sp. L. & Asteraceae & A-B & $\mathrm{C} 3$ & & 1 & & \\
\hline Carduus acanthoides L. & Asteraceae & A & $\mathrm{C} 3$ & & & 2 & \\
\hline Carduus pycnocephalus L. & Asteraceae & A & $\mathrm{C} 3$ & & 1 & & 2 \\
\hline Carduus sp. L & Asteraceae & A & $\mathrm{C} 3$ & 3 & 1 & 6 & \\
\hline Carduus tenuiflorus Curtis & Asteraceae & A & $\mathrm{C} 3$ & & & 2 & \\
\hline Carthamus lanatus L. & Asteraceae & A & $\mathrm{C} 3$ & 3 & & 4 & \\
\hline Carthamus sp. L. & Asteraceae & A & $\mathrm{C} 3$ & & 4 & & \\
\hline Centaurea sp. L. & Asteraceae & A & $\mathrm{C} 3$ & & 1 & 6 & \\
\hline Centaurium pulchellum (Sw.) Druce & Gentianaceae & A & $\mathrm{C} 3$ & 15 & 9 & 2 & \\
\hline Centunculus minimus L. & Primulaceae & A & $\mathrm{C} 3$ & 25 & 28 & 55 & 9 \\
\hline Cerastium sp. L. & Caryophyllaceae & A & $\mathrm{C} 3$ & & 3 & 15 & \\
\hline Cerastium glomeratum Thuill. & Caryophyllaceae & A & $\mathrm{C} 3$ & 11 & 6 & 2 & \\
\hline Cirsium vulgare (Savi) Ten. & Asteraceae & A & $\mathrm{C} 3$ & 9 & 30 & 60 & 17 \\
\hline Echium plantagineum L. & Boraginaceae & A & $\mathrm{C} 3$ & & 3 & 9 & \\
\hline Erodium sp. L’Hér ex Aiton & Geraniaceae & A-B & $\mathrm{C} 3$ & & & & 2 \\
\hline Fimbristylis dichotoma (L.) Vahl & Cyperaceae & $\mathrm{P}$ & $\mathrm{C} 4$ & & & 7 & \\
\hline Hypochoeris glabra $\mathrm{L}$. & Asteraceae & A & $\mathrm{C} 3$ & & 3 & 2 & 7 \\
\hline Hypochoeris radicata $\mathrm{L}$. & Asteraceae & A & $\mathrm{C} 3$ & & 7 & 35 & 10 \\
\hline Lotus corniculatus L. & Fabaceae & $\mathrm{P}$ & $\mathrm{C} 3$ & & & 24 & \\
\hline Lotus subbiflorus Lag. & Fabaceae & A & $\mathrm{C} 3$ & 1 & 11 & 35 & 2 \\
\hline Medicago lupulina L. & Fabaceae & A & $\mathrm{C} 3$ & & 1 & 4 & \\
\hline Medicago sp. L & Fabaceae & A & $\mathrm{C} 3$ & & 4 & 7 & \\
\hline Mentha pulegium L. & Lamiaceae & $\mathrm{P}$ & $\mathrm{C} 3$ & & 7 & 6 & \\
\hline Parentucellia viscosa (L.) Caruel & Orobanchaceae & A & C3 & & & 2 & \\
\hline Plantago lanceolata $\mathrm{L}$. & Plantaginaceae & $\mathrm{P}$ & $\mathrm{C} 3$ & & & 2 & \\
\hline Polycarpon tetraphyllum (L.) L. & Caryophyllaceae & A & $\mathrm{C} 3$ & & & & 5 \\
\hline Senecio madagascariensis Poir & Asteraceae & $\mathrm{P}$ & $\mathrm{C} 3$ & & & 2 & \\
\hline Silene gallica L. & Caryophyllaceae & A & $\mathrm{C} 3$ & & 6 & 18 & 8 \\
\hline Sonchus asper (L.) Hill & Asteraceae & A-B & $\mathrm{C} 3$ & & 1 & 7 & \\
\hline
\end{tabular}


Apéndice. Continuación.

Appendix. Continuation.

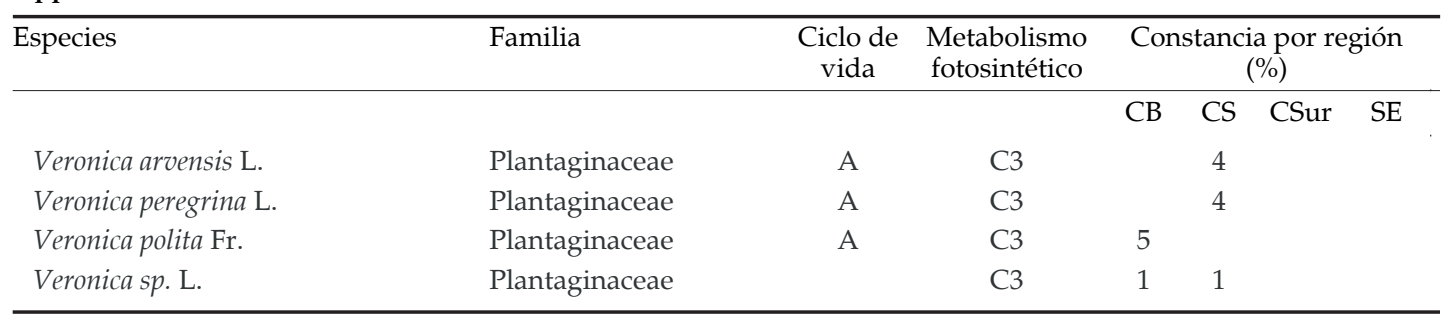

